Solar Physics

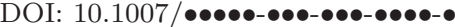

\title{
Slow Mode Oscillations and Damping of Hot Solar Coronal Loops
}

Solar Physics

\author{
A. Abedini ${ }^{1} \cdot$ H. Safari ${ }^{2} \cdot$ S. Nasiri ${ }^{2}$ \\ (C) Springer $\bullet \bullet \bullet \bullet$
}

\begin{abstract}
The effect of temperature inhomogeneity on the periods, their ratios (fundamental vs. first overtone), and the damping times of the standing slow modes in gravitationally stratified solar coronal loops are studied. The effects of optically thin radiation, compressive viscosity, and thermal conduction are considered. The linearized one-dimensional magnetohydrodynamic (MHD) equations (under low- $\beta$ condition) were reduced to a fourth-order ordinary differential equation for the perturbed velocity. The numerical results indicate that the periods of non-isothermal loops (i.e. temperature increases from the loop base to apex) are smaller compared to those of isothermal loops. In the presence of radiation, viscosity, and thermal conduction, an increase in the temperature gradient is followed by a monotonic decrease in the periods (compared with the isothermal case), while the period ratio turns out to be a sensitive function of the gradient of the temperature and the loop lengths. We verify that radiative dissipation is not a main cooling mechanism of both isothermal and non-isothermal hot coronal loops and has a small effect on the periods. Thermal conduction and compressive viscosity are primary mechanisms in the damping of slow modes of the hot coronal loops. The periods and damping times in the presence of compressive viscosity and/or thermal conduction dissipation are consistent with the observed data in specific cases. By tuning the dissipation parameters, the periods and the damping times could be made consistent with the observations in more general cases.
\end{abstract}

Keywords: Sun, corona; Sun, magnetic fields; Sun, oscillations

\section{Introduction}

Through SOHO and TRACE observations, propagating slow magnetoacoustic waves have been found in coronal plumes, loop footpoints, above sunspots and

\footnotetext{
1 Department of Physics, Institute for Advanced Studies in Basic Sciences, Zanjan, 45137-66731, I. R. Iran

2 Department of Physics, University of Zanjan, University Blvd., 45371-38791, Zanjan, I. R. Iran email: safari@znu.ac.ir
} 
even non-sunspot regions (e.g., Ofman et al., 1997; DeForest and Gurman, 1998; Berghmans and Clette, 1999; De Moortel et al., 2000; ÓShea et al., 2002; Brynildsen et al., 2002; Marsh et al., 2003). Standing longitudinal slow waves with strong damping and large Doppler-shift oscillations have been detected in hot postflare loops recorded by SOHO/SUMER. In the cooler loops, these waves were observed by the EUV imaging spectrometer Hinode/EIS (Srivastava and Dwived, 2010). These oscillations have a phase shift of about one-quarter period between velocity and intensity. The periods and damping times of the standing slow waves are in the ranges of $8.6-32.3 \mathrm{~min}$ and $3.1-42.3 \mathrm{~min}$, respectively (Kliem et al., 2002; Wang et al., 2002a, 2002b, 2003, 2005; Banerjee et al., 2007; Erdélyi et al., 2008). The effect of energy dissipation on the slow waves through thermal conduction, compressive viscosity, radiative cooling, and heating on the periods, period ratio, and damping times has formed the focus of recent studies. Ofman and Wang (2002), for instance, studied the oscillations and damping of standing slow modes in the isothermal loops. They found that thermal conduction is the dominant dissipation mechanism. Taking into account the effects of thermal conduction and compressive viscosity, De Moortel and Hood (2003) investigated both propagating and standing slow magnetoacoustic waves in homogeneous corona loops. They found a minimum damping time that can be obtained by thermal conduction alone. However, for stronger dissipation an additional mechanism such as viscosity has to be added. Sigalotti et al. (2007) studied the dissipation of standing slow modes in hot, isothermal loops by integrating the effects of gravitational stratification, thermal conduction, compressive viscosity, radiative cooling, and heating in their study. They concluded that thermal conduction and compressive viscosity are the main sources of the wave damping. Pandey and Dwivedi (2006) have shown that separate effects of thermal conduction and viscosity in isothermal loops are not sufficient to explain the observed damping times of the oscillations. Only with the combined effect of thermal conduction and viscosity the results are consistent with the observations. Moreover, Taroyan et al. (2005) investigated the effect of temperature inhomogeneity on the dissipation of standing slow waves through considering thermal conduction and optically thin radiative losses. They found that the damping time of the isothermal loops is proportional to the wave period and the oscillations are rapidly damped mainly by thermal conduction.

The ratio between the period of the fundamental mode $\left(p_{1}\right)$ and the first overtone $\left(p_{2}\right), p_{1} /\left(2 p_{2}\right)$, for both the fast kink mode and the slow longitudinal mode is a useful seismological indicator. Departure of this ratio from unity is a consequence of the density, pressure, temperature, magnetic field (both radial and longitudinal structures), heating functions and the dissipation mechanisms ( Díaz et al., 2006; Dymova and Ruderman, 2006; Donnelly et al., 2006; Erdélyi and Verth, 2007; Safari et al., 2007; Verth et al., 2007; Ruderman et al., 2008; McEwan et al., 2008; Andries et al., 2009; Fathalian and Safari, 2010). The results of the numerical modeling of the oscillations allow a comparison between the model and the observational results (McEwan et al., 2006; Abedini and Safari, 2011). Macnamara and Roberts (2010) studied the effects of thermal conduction and compressive viscosity on the slow mode of the isothermal loops, and concluded that the effect of thermal conduction on the period ratio is negligible. 
In line with the above-mentioned studies, in this study, we will investigate the dissipation of the standing slow MHD modes in the hot coronal loops taking into account gravitational stratification, inhomogeneity of temperature, thermal conduction, compressive viscosity, heating, and optically thin radiative losses.

For such a purpose, the linearized one dimensional magnetohydrodynamic (MHD) equations are reduced to a fourth-order differential equation for the perturbed velocity. The paper is organized as follows. In Section 2, we present a brief description of the models and equations. In Section 3, the differential equation is solved numerically based on the boundary value problem solver (finite difference code) for different cases. Finally, conclusions are drawn in Section 4.

\section{Description of the Model and Equations}

Coronal loops are modeled as a magnetic flux tube of length $2 L$ with a shape of half-circle and symmetrical around the apex $(z=0)$, with a constant magnetic field along the loop length. The footpoints are fixed in the chromosphere. The equilibrium plasma flow is ignored, $\mathbf{v}_{0}=0$. The projection of the gravity acceleration on the tangent to the loop (half-circle) is equal to $g \sin \pi z / 2 L$. The equilibrium temperature is considered as a function of $z$,

$$
T_{0}(z)=T_{0}(L)\left[1+(\lambda-1) \cos \frac{\pi z}{2 L}\right], \quad-L \leq z \leq L, \quad \lambda=\frac{T_{0}(0)}{T_{0}(L)},
$$

where $T_{0}(0)$ and $T_{0}(L)$ are the temperatures at the apex and at the base, respectively. Under the ideal gas law as well as in the low- $\beta$ plasma, the equilibrium density and pressure are given by

$$
\begin{aligned}
& \rho_{0}(z)=\rho_{0}(L)\left[1+(\lambda-1) \cos \frac{\pi z}{2 L}\right]^{-\left(\frac{N}{\alpha_{T}}+1\right)}, \\
& p_{0}(z)=p_{0}(L)\left[1+(\lambda-1) \cos \frac{\pi z}{2 L}\right]^{-\frac{N}{\alpha_{T}}}, \alpha_{T}=\frac{\pi(\lambda-1)}{2},
\end{aligned}
$$

where $N=L / \Lambda_{0}(L)$ and the pressure scale height is defined by $\Lambda_{0}=p_{0} / \rho_{0} g$. The energy terms, the constant heating rate $\left(H=H_{0}\right)$, the conductive heating $\left(E_{\mathrm{C}}\right)$, the radiative cooling $\left(E_{\mathrm{R}}\right)$, and the compressive viscous heating $\left(E_{\eta}\right)$ all are given by

$$
E_{\mathrm{C}}=\frac{\partial}{\partial z}\left(k_{\|} \frac{\partial T}{\partial z}\right), E_{\mathrm{R}}=\chi \rho^{2} T^{\alpha}, E_{\eta}=\frac{4}{3} \eta\left(\frac{\partial v}{\partial z}\right)^{2},
$$

in which $k_{\|}=10^{-11} T^{5 / 2}\left(\mathrm{Wm}^{-1} \mathrm{~K}^{-1}\right)$ and $\eta=10^{-17} T^{5 / 2}\left(\mathrm{~kg} \mathrm{~m}^{-1} \mathrm{~s}^{-1}\right)$ are the coefficient of thermal conduction along the magnetic field and the coefficient of compressive viscosity, respectively (Braginskii, 1965; Hildner, 1974; Priest, 1982). The parameters $\alpha$ and $\chi$ are $\alpha=-1$ and $\chi=10^{24} \mathrm{Wm}^{3} \mathrm{~kg}^{-2} \mathrm{~K}$, respectively, for the temperature range of $0.8-10 \mathrm{MK}$ (Sigalotti et al., 2007). In the equilibrium state, we get $H_{0}+E_{0 \mathrm{C}}+E_{0 \eta}=E_{0 \mathrm{R}}$. For the convenience of our analysis, the 
dimensionless parameters are defined as

$$
\begin{aligned}
& \bar{t}=t / \tau_{\mathrm{s}}, \bar{z}=\frac{z}{L}, \bar{v}=\frac{v}{c_{\mathrm{s}}(L)}, \bar{T}=\frac{T}{T_{0}(L)}, \bar{p}=\frac{p}{p_{0}(L)}, \bar{\rho}=\frac{\rho}{\rho_{0}(L)}, \\
& \bar{\eta}=\frac{\eta}{\eta_{0}(L)}, \overline{k_{\|}}=\frac{k_{\|}}{k_{0||}(L)}, r=\frac{(\gamma-1) \tau \chi \rho_{0}^{2}(L) T_{0}^{-1}(L)}{\gamma p_{0}(L)}, \\
& \epsilon=\frac{1}{\operatorname{Re}}=\frac{\eta_{0}(L) \tau_{\mathrm{s}}}{\rho_{0}(L) L^{2}}, \quad d=\frac{(\gamma-1) k_{0||}(L) T_{0}(L) \rho_{0}(L)}{\gamma^{2} p_{0}^{2}(L) \tau_{\mathrm{s}}},
\end{aligned}
$$

where $c_{\mathrm{s}}^{2}(L)=\gamma P_{0}(L) / \rho_{0}(L)$ is the adiabatic sound speed, $\tau_{\mathrm{s}}=L / c_{\mathrm{s}}$ is the sound crossing time from the loop footpoints to the apex, and Re is the Reynolds number. The dimensionless parameters $d, r$, and $\epsilon$ are called the thermal ratio, radiation ratio, and the compressive viscosity ratio, respectively (e.g., De Moortel and Hood, 2003, 2004). In hot coronal plasma conditions, the values of $r, \epsilon$, $d$ and $\tau_{\mathrm{s}}$ are tabulated in Table 1 .

Table 1. Dimensionless ratios and the sound crossing time as functions of the loop length $(L)$ and the apex temperature $\left(T_{0}(L)\right)$.

\begin{tabular}{cccccc}
\hline$T_{0}(L)[\mathrm{MK}]$ & $L[\mathrm{Mm}]$ & $r \times 10^{3}$ & $\epsilon$ & $d$ & $\tau_{\mathrm{s}}[\mathrm{min}]$ \\
\hline 6 & $20-220$ & $0.05-0.53$ & $0.02-0.21$ & $0.54-6.0$ & $0.88-9.64$ \\
\hline 10 & $20-220$ & $0.01-0.11$ & $0.08-0.83$ & $2.18-24.0$ & $0.69-7.65$ \\
\hline
\end{tabular}

In the low- $\beta$ condition, the linearized non-ideal MHD equations are

$$
\begin{aligned}
& \frac{\partial \rho_{1}}{\partial t}+\rho_{0} \frac{\partial v_{1}}{\partial z}+\frac{\partial \rho_{0}}{\partial z} v_{1}=0, \\
& \rho_{0} \frac{\partial v_{1}}{\partial t}=-\frac{\partial p_{1}}{\gamma \partial z}+\frac{N \sin \left(\frac{\pi z}{2}\right) \rho_{1}}{\gamma}+\eta_{0} \varepsilon \frac{4}{3} \frac{\partial^{2} v_{1}}{\partial z^{2}}, \\
& \frac{p_{1}}{p_{0}}=\frac{\rho_{1}}{\rho_{0}}+\frac{T_{1}}{T_{0}}, \\
& \frac{\partial p_{1}}{\partial t}+v_{1} \frac{\partial p_{0}}{\partial z}+\gamma p_{0} \frac{\partial v_{1}}{\partial z}= \\
& \quad \gamma\left(-2 r E_{0 \mathrm{R}} \frac{\rho_{1}}{\rho_{0}}+r E_{0 \mathrm{R}} \frac{T_{1}}{T_{0}}+d \frac{\partial}{\partial z}\left(k_{0 \| \mid} \frac{\partial T_{1}}{\partial z}+k_{1 \|} \frac{\partial T_{0}}{\partial z}\right)\right) .
\end{aligned}
$$

As we note here, all the quantities in Equations (5)-(7) are dimensionless (bars on dimensionless quantities have been dropped). The perturbed quantities are assumed to be of the form $f_{1}(z, t)=f_{1}(z) \exp (\nu t)$. After some algebra, Equations (5)-(7) reduce to a fourth-order differential equation as

$$
\mathcal{A}(z, r, \epsilon, d, \nu) \frac{d^{4} v_{1}}{d z^{4}}+\mathcal{B}(z, r, \epsilon, d, \nu) \frac{d^{3} v_{1}}{d z^{3}}+\mathcal{C}(z, r, \epsilon, d, \nu) \frac{d^{2} v_{1}}{d z^{2}}
$$




$$
+\mathcal{D}(z, r, \epsilon, d, \nu) \frac{d v_{1}}{d z}+\mathcal{E}(z, r, \epsilon, d, \nu)=0 .
$$

Coefficients $\mathcal{A}, \mathcal{B}, \mathcal{C}, \mathcal{D}$, and $\mathcal{E}$ are given in the Appendix.

The boundary conditions are

- The footpoints $(z= \pm 1)$ are expected to be the nodes, i.e., $\left.v\right|_{\text {footpoints }}=0$.

- Near the apex, $z \rightarrow 0$, all the coefficients in Equations (5)-(7) are constants and the solutions are expected to be $v(z, t) \sim \exp [-i(\omega t-k z)]$.

In the remainder of this paper, Equation (8) is solved for five various cases by imposing the appropriate boundary conditions.

\section{Solutions to Equation (8)}

In all of the following cases, the loops are considered to be gravitationally stratified.

\subsection{Case 1: $d=r=\epsilon=0, N \neq 0$}

In the absence of all dissipation terms, Equation (8) reduces to a second order ordinary differential equation. The resultant equation is solved numerically. In Figure 1, the fundamental and the first overtone modes and their ratios, $p_{1} /\left(2 p_{2}\right)$, are plotted versus $L$. As shown in the figure, in the case of adiabatic and isothermal loops $(\lambda \rightarrow 1)$, the fundamental and first overtone periods are in the ranges of 3-32 and 1-18 min, respectively. The results are well consistent with the previous studies (Curdt et al., 2002; McEwan et al., 2006; Sigalotti et al., 2007; Abedini and Safari, 2011).

We see that, by increasing $\lambda$ from 1 to 2 the periods and their ratio decrease; yet, the changes in the fundamental periods are larger than those of the overtone periods. For typical loop lengths of 20-200 Mm, after adding the temperature gradient $(\lambda=2)$ the changes in the periods is found to be about a few minutes $(\leq 10)$.

3.2. Case 2: $d=\epsilon=0, r \neq 0, N \neq 0$

In the presence of optically thin radiation (i.e., $\epsilon=d=0, r \neq 0$ ), similar to the previous case, we have a second order differential equation. The numerical solutions to the resultant equation are plotted in Figures 2 and 3. By comparing Figures 1 and 2, we find that the fundamental and the first overtone modes have changed about one percent. Therefore we conclude that the radiation does not change the periods and their ratios significantly. In Figure 3, the damping time and damping quality (damping per period, $\tau_{\mathrm{d}} / p=\operatorname{Re}(\omega) / 2 \pi \operatorname{Im}(\omega)$ ) are plotted versus $L$ for different $T_{\text {base }}$ and $\lambda$. As shown in the figures, the damping time is of the order of $10^{6} \mathrm{~min}$ and the damping quality is of the order of $10^{5}$. This case lies in the weak damping regime $\left(\tau_{\mathrm{d}} / p \geq 2\right)$. By increasing $\lambda$ from 1 to 2 , the damping time and damping quality increase. We arrive at the conclusion that, in the hot coronal loop, radiation is not a primary mechanism for the damping of the slow modes. 
3.3. Case 3: $r=d=0, \epsilon \neq 0, N \neq 0$

Next, the effects of compressive viscosity on the oscillations and damping of the loops are investigated. The numerical solutions are shown in Figures 4 and 5. By comparing Figures 1 and 4 we see the following: Compressive viscosity introduces a cutoff frequency on the oscillations as a function of both inhomogeneity parameter $\lambda$ and loop length $L$ (see, e.g., Sigalotti et al., 2007). The effect of compressive viscosity on the periods for a typical loop length of $20-200 \mathrm{Mm}$ is a few percent $(\leq 2 \%)$ increase compared to case 1 . By increasing inhomogeneity in the temperature $(\lambda)$ and decreasing the loop length, the ratio $\left(p_{1} /\left(2 p_{2}\right)\right)$ deviates from unity. At higher temperatures $(\geq 6 \mathrm{MK})$ and non-isothermal loops, viscous dissipation is one of the primary damping mechanisms. Indeed, viscosity is one of the main factors for the strong damping in the short and non-isothermal loops and is consistent with the observational data.

In the presence of the temperature gradient, the damping of the first overtone is stronger than that of the fundamental mode. Unlike radiation (case 2), if $\lambda$ is increased from 1 to 2 in the presence of viscosity, the damping time and damping quality decrease. We conclude that, for non-isothermal loops in the presence of viscosity and gravity, the computed damping times are consistent with the SUMER observations (Wang et al., 2003, 2005).

\subsection{Case $4: d \neq 0, r=\epsilon=0, N \neq 0$}

In the presence of thermal conduction, the periods decrease slightly if the temperature increases (Figure 6). However, by increasing inhomogeneity in the temperature ( $\lambda$ from 1 to 2 ), the periods and their ratio decrease considerably. In other words, the decrease in the period is more sensitive to $\lambda$ than the temperature itself. The effect of thermal conduction on the periods compared to the stratified isothermal loops (case $1, \lambda \rightarrow 1$ ) is about $18 \mathrm{~min}$. For $\lambda=2$ the increase in the periods is about $8 \mathrm{~min}$. The deviation in the period ratio from unity increases with increasing $L$ and $\lambda$ (for $T_{\text {base }}=6 \mathrm{MK}$ ). The deviation of the damping quality from unity in isothermal loops decreases with increasing $L$ for short loops (Figure 7). However, if the loop length is further increased, the damping quality of non-isothermal loops becomes larger than that of the isothermal case.

There is a negative relationship between the damping time and temperature in the case of non-isothermal loops: An increase in the temperature brings about a decrease in the damping time. On the other hand, there is a positive relationship between the damping time and the loop length. We see that, in the presence of thermal conduction, the damping of both isothermal and non-isothermal long loops are in the strong regime $\left(\tau_{\mathrm{d}} / p \leq 1\right)$.

3.5. Case $5: d \neq 0, \epsilon \neq 0, r \neq 0, N \neq 0$

In the most general case which includes heat conduction, compressive viscosity, and optically thin radiation, Equation (8) must be solved. The numerical results are presented in Figures 8 and 9. In Figure 8, periods and their ratios are plotted 
versus $L$. Compared with the case of the adiabatic loops (Figure 1), the periods increase noticeably. Periods with $T_{\text {base }}=6$ and $10 \mathrm{MK}$ are 2-50 min and 1-45 min, respectively (for isothermal loops). By increasing $\lambda$ from 1 to 2 , the periods decrease about $20 \mathrm{~min}$.

In the presence of all dissipation mechanisms and temperature gradient, the damping time and damping quality are consistent with the observed values (Banerjee et al., 2007; Kliem et al., 2002; Wang et al., 2002a, 2002b, 2003, 2005).

\section{Summary}

The coronal loop has been modeled as a half-circle magnetic flux tube with a uniform magnetic field along the loop axis. The effect of the inhomogeneity of the temperature on the periods, the period ratio, and damping times of standing slow modes in the gravitationally stratified coronal loops was investigated in this study. The effects of optically thin radiation, compressive viscosity, and thermal conduction were considered. The linearized one-dimensional MHD equations were reduced to a fourth-order ordinary differential equation for the perturbed velocity. Under the solar coronal conditions (low- $\beta$ plasma) the resultant equation was solved numerically. Moreover, the oscillations and the damping of various flux tube models in the presence of dissipation mechanisms were compared with the oscillations and damping of adiabatic and isothermal loops. The following shows the main results:

- In a gravitationally stratified loop, the periods and their ratio (fundamental vs. first overtone) are sensitive functions of the temperature inhomogeneity.

- In hot corona loops ( $T \geq 6 \mathrm{MK})$, the effect of optically thin radiation on the periods and damping of the oscillations is negligible for both isothermal and non-isothermal cases.

- In the presence of compressive viscosity, the damping times significantly change but viscous dissipation is more effective in shorter and hotter loops. Compressive viscosity introduces a cutoff frequency on the oscillations.

In the case of non-isothermal and hotter loops the computed damping times agree with the observed data.

- Thermal conduction changes the periods more significantly than other dissipation mechanisms, and the damping of oscillations is in the weak regime $\left(\tau_{\mathrm{d}} / p \geq 2\right)$. We noted that thermal conduction is a function of the temperature gradient and depends on higher derivatives, and this is the reason for bringing more changes in the periods in addition to the changes due to inhomogeneity in the temperature.

- In the most general case where optically thin radiation, compressive viscosity, and thermal conduction are all considered, the periods are positively correlated with the loop length and negatively correlated with the temperature. The damping time and damping quality are complex functions of both loop length $L$ and inhomogeneity parameter $\lambda$. We also found that in the isothermal gravitationally stratified loops, thermal conduction and compressive viscosity are needed to reproduce the observed periods and 
Abedini et al.

damping times. In the presence of the temperature gradient in the gravitationally stratified non-isothermal loops, the periods and the damping times resulted by compressive viscosity and/or thermal conduction dissipation, are consistent with the observed data in special cases. However, by tuning the dissipation parameters, the periods and the damping times could be made in good agreement with the observations in more general cases.

Acknowledgements The authors thank the anonymous referee for very helpful comments and suggestions.

\section{Appendix}

Coefficient $\mathcal{A}, \mathcal{B}, \mathcal{C}, \mathcal{D}$, and $\mathcal{E}$ in Equation (8) are:

$$
\begin{aligned}
& T_{0}(z)=\left[1+(\lambda-1) \cos \left(\frac{\pi z}{2}\right], \rho_{0}(z)=T_{0}(z)^{-\left(\frac{N}{\alpha_{T}}+1\right)}, p_{0}(z)=T_{0}(z)^{-\frac{N}{\alpha_{T}}},\right. \\
& g=\sin \left(\frac{\pi z}{2}\right), k_{0||}=T_{0}^{5 / 2}(z), \eta_{0}(z)=T_{0}^{5 / 2}(z), \\
& f(z)=\gamma d k_{0 \| \mid}\left[-\frac{T_{0} p_{0}^{\prime \prime}}{p_{0}^{2}}+\frac{2 T_{0} p_{0}^{\prime 2}}{p_{0}^{3}}-\frac{7 T_{0}^{\prime} p_{0}^{\prime}}{p_{0}^{2}}+\frac{35 T_{0}^{\prime 2}}{4 p_{0} T_{0}}+\frac{7 T_{0}^{\prime \prime}}{2 p_{0}}\right]+r \gamma \rho_{0} T_{0}^{-2} \\
& \mathcal{A}(r, \epsilon, d, z)=\gamma d k_{0||}\left[\frac{4 \gamma \eta_{0} \epsilon \nu}{3 \rho_{0}}+T_{0}\right] \\
& \mathcal{B}(r, \epsilon, d, z)=\gamma d k_{0 \|}\left[\left(\frac{19 T_{0}^{\prime}}{2 p_{0}}-\frac{2 T_{0} p_{0}^{\prime}}{p_{0}^{2}}-\frac{\rho_{0}^{\prime}}{\rho_{0}^{2}}\right) \frac{4 \gamma \eta_{0} \epsilon}{3} \nu+\frac{4 \gamma \eta_{0}^{\prime} \epsilon}{\rho_{0}} \nu\right] \\
& +\gamma d k_{0 \| \mid}\left[-N g+\frac{T_{0} \rho_{0}^{\prime}}{\rho_{0}}+\frac{21 T_{0}^{\prime}}{2}-\left(T_{0}+\frac{4 \gamma \eta_{0} \epsilon}{3 \rho_{0}} \nu\right) \frac{f(z)^{\prime}}{(f(z)-\nu)}\right] \\
& \mathcal{C}(r, \epsilon, d, z)=+\gamma d k_{0||}\left[\left(\frac{35 T_{0}^{\prime 2}}{2 T_{0} p_{0}}+\frac{7 T_{0}^{\prime \prime}}{p_{0}}-\frac{14 T_{0}^{\prime} p_{0}^{\prime}}{p_{0}^{2}}-\frac{2 T_{0} p_{0}^{\prime \prime}}{p_{0}^{2}}+\frac{4 T_{0} p_{0}^{\prime 2}}{p_{0}^{3}}\right) \gamma \eta_{0} \epsilon \frac{4}{3} \nu\right] \\
& +\gamma d k_{0 \| \mid}\left(\frac{19 T_{0}^{\prime}}{2 p_{0}}-\frac{2 T_{0} p_{0}^{\prime}}{p_{0}^{2}}-\frac{\rho_{0}^{\prime}}{\rho_{0}^{2}}\right)\left[\gamma \eta_{0}^{\prime} \epsilon \frac{8}{3} \nu-N g \rho_{0}\right] \\
& +\gamma d k_{0 \| \mid}\left[\frac{3 T_{0} \rho_{0}^{\prime \prime}}{\rho_{0}}+\frac{105 T_{0}^{\prime 2}}{4 T_{0}}+\frac{21 T_{0}^{\prime \prime}}{2}+\frac{21 T_{0}^{\prime} \rho_{0}^{\prime}}{2 \rho_{0}}-\frac{3 T_{0} \rho_{0}^{\prime 2}}{\rho_{0}^{2}}-2 N g^{\prime}-\frac{3 N g \rho_{0}^{\prime}}{\rho_{0}}-\gamma \nu^{2}\right] \\
& \left.+\gamma d k_{0 \| \mid}\left[N g-7 T_{0}^{\prime}-\frac{T_{0} \rho_{0}^{\prime}}{\rho_{0}}+\left(-\frac{7 T_{0}^{\prime}}{p_{0}}+\frac{2 T_{0} p_{0}^{\prime}}{p_{0}^{2}}\right) \gamma \eta_{0} \epsilon \frac{4}{3} \nu-\gamma \eta_{0}^{\prime} \epsilon \frac{8}{3 \rho_{0}} \nu\right)\right] \frac{f(z)^{\prime}}{(f(z)-\nu)} \\
& +3 r \gamma \rho_{0}^{2} T_{0}^{-1}+(f(z)-\nu) \frac{4 \gamma \eta_{0} \epsilon}{3} \nu-\gamma p_{0} \nu \\
& \left.\mathcal{D}(r, \epsilon, d, z)=+\gamma d k_{0||}\left[\frac{35 T_{0}^{\prime 2}}{2 T_{0} p_{0}}+\frac{7 T_{0}^{\prime \prime}}{p_{0}}-\frac{14 T_{0}^{\prime} p_{0}^{\prime}}{p_{0}^{2}}-\frac{2 T_{0} p_{0}^{\prime \prime}}{p_{0}^{2}}+\frac{4 T_{0} p_{0}^{\prime 2}}{p_{0}^{3}}\right)\left(\gamma \eta_{0}^{\prime} \epsilon \frac{4}{3} \nu-N g \rho_{0}\right)\right] \\
& +\gamma d k_{0 \| \mid}\left[\frac{21 T_{0}^{\prime} \rho_{0}^{\prime \prime}}{\rho_{0}}+\frac{3 T_{0} \rho_{0}^{\prime \prime \prime}}{\rho_{0}}-\frac{9 T_{0} \rho_{0}^{\prime} \rho_{0}^{\prime \prime}}{\rho_{0}^{2}}-\frac{21 T_{0}^{\prime} \rho_{0}^{\prime 2}}{\rho_{0}^{2}}+\frac{6 T_{0} \rho_{0}^{\prime 3}}{\rho_{0}^{3}}+\frac{21 \rho_{0}^{\prime} T_{0}^{\prime \prime}}{2 \rho_{0}}\right. \\
& \left.+\frac{105 T_{0}^{\prime} T_{0}^{\prime \prime}}{4 T_{0}}+\frac{105 T_{0}^{\prime 2} \rho_{0}^{\prime}}{4 \rho_{0} T_{0}}+\frac{7 T_{0}^{\prime \prime \prime}}{2}+\frac{105 T_{0}^{\prime 3}}{8 T_{0}^{2}}\right] \\
& +\gamma d k_{0||}\left[\frac{4 \gamma \eta_{0}^{\prime \prime \prime} \epsilon}{3 \rho_{0}} \nu-N g^{\prime \prime}-\frac{4 N g^{\prime} \rho_{0}^{\prime}}{\rho_{0}}-\frac{3 N g \rho_{0}^{\prime \prime}}{\rho_{0}}-\frac{2 \gamma \rho_{0}^{\prime} \nu^{2}}{\rho_{0}}\right] \\
& +\gamma d k_{0 \|||}\left[\left(\frac{19 T_{0}^{\prime}}{2 p_{0}}-\frac{2 T_{0} p_{0}^{\prime}}{p_{0}^{2}}-\frac{\rho_{0}^{\prime}}{\rho_{0}^{2}}\right)\left(\gamma \eta_{0}^{\prime \prime} \epsilon \frac{4}{3} \nu-N g^{\prime} \rho_{0}-2 N g \rho_{0}^{\prime}-\gamma \rho_{0} \nu^{2}\right)\right] \\
& -\gamma d k_{0 \|}\left[\left(\frac{7 T_{0}^{\prime}}{p_{0}}-\frac{2 T_{0} p_{0}^{\prime}}{p_{0}^{2}}\right)\left(\frac{4 \gamma \eta_{0}^{\prime} \epsilon \nu}{3}-N g \rho_{0}\right)+\left(\frac{4 \gamma \eta_{0}^{\prime \prime} \epsilon \nu}{3 \rho_{0}}-N g^{\prime}-\frac{2 N g \rho_{0}^{\prime}}{\rho_{0}}-\gamma \nu^{2}\right)\right] \frac{f(z)^{\prime}}{(f(z)-\nu)} \\
& -\gamma d k_{0 \| \mid}\left[\left(\frac{2 T_{0} \rho_{0}^{\prime \prime}}{\rho_{0}}+\frac{35 T_{0}^{\prime 2}}{4 T_{0}}+\frac{7 T_{0}^{\prime \prime}}{2}+\frac{7 T_{0}^{\prime} \rho_{0}^{\prime}}{\rho_{0}}-\frac{2 T_{0} \rho_{0}^{\prime 2}}{\rho_{0}^{2}}\right) \frac{f(z)^{\prime}}{(f(z)-\nu)}\right] \\
& +3 r \gamma\left[\left(3 \rho_{0}^{\prime} \rho_{0} T_{0}^{-1}-\rho_{0}^{2} T_{0}^{\prime} T_{0}^{-2}\right)-\rho_{0}^{2} T_{0}^{-1} \frac{f(z)^{\prime}}{(f(z)-\nu)}\right] \\
& -(1+\gamma) p_{0}^{\prime} \nu+\gamma p_{0} \nu \frac{f(z)^{\prime}}{(f(z)-\nu)}+(f(z)-\nu)\left(\frac{4 \gamma \eta_{0}^{\prime} \epsilon}{3} \nu-N g \rho_{0}\right) \\
& \mathcal{E}(r, \epsilon, d, z)=-\gamma d k_{0 \| \mid[}\left[\left(\frac{19 T_{0}^{\prime}}{2 p_{0}}-\frac{2 T_{0} p_{0}^{\prime}}{p_{0}^{2}}-\frac{\rho_{0}^{\prime}}{\rho_{0}^{2}}\right)\left(N g \rho_{0}^{\prime \prime}+N g^{\prime} \rho_{0}^{\prime}+\gamma \rho_{0}^{\prime} \nu^{2}\right)\right. \\
& -\gamma d k_{0||}\left[\frac{35 T_{0}^{\prime 2}}{2 T_{0} p_{0}}+\frac{7 T_{0}^{\prime \prime}}{p_{0}}-\frac{14 T_{0}^{\prime} p_{0}^{\prime}}{p_{0}^{2}}-\frac{2 T_{0} p_{0}^{\prime \prime}}{p_{0}^{2}}+\frac{4 T_{0} p_{0}^{\prime 2}}{p_{0}^{3}}\right]\left[N g \rho_{0}^{\prime}+\gamma \rho_{0} \nu^{2}\right] \\
& +\gamma d k_{0 \| \mid}\left[-\frac{63 T_{0}^{\prime} \rho_{0}^{\prime} \rho_{0}^{\prime \prime}}{2 \rho_{0}^{2}}+\frac{21 T_{0}^{\prime} \rho_{0}^{\prime 3}}{\rho_{0}^{3}}+\frac{105 T_{0}^{\prime 3} \rho_{0}^{\prime}}{8 \rho_{0} T_{0}^{2}}+\frac{12 T_{0} \rho_{0}^{\prime 2} \rho_{0}^{\prime \prime}}{\rho_{0}^{3}}-\frac{6 T_{0} \rho_{0}^{\prime 4}}{\rho_{0}^{4}}-\frac{105 T_{0}^{\prime 2} \rho_{0}^{\prime 2}}{4 \rho_{0}^{2} T_{0}}+\frac{105 T_{0}^{\prime 2} \rho_{0}^{\prime \prime}}{4 \rho_{0} T_{0}}\right.
\end{aligned}
$$


Slow Mode Oscillations of Coronal Loops ...

$$
\begin{aligned}
& \left.-\frac{3 T_{0} \rho_{0}^{\prime \prime 2}}{\rho_{0}^{2}}-\frac{4 T_{0} \rho_{0}^{\prime} \rho_{0}^{\prime \prime \prime}}{\rho_{0}^{2}}+\frac{21 T_{0}^{\prime} \rho_{0}^{\prime \prime \prime}}{2 \rho_{0}}+\frac{\rho_{0}^{\prime \prime \prime} T_{0}}{\rho_{0}}-\frac{21 \rho_{0}^{\prime} T_{0}^{\prime \prime} \rho_{0}^{\prime}}{2 \rho_{0}^{2}}+\frac{105 T_{0}^{\prime} T_{0}^{\prime \prime} \rho_{0}^{\prime}}{4 T_{0} \rho_{0}}+\frac{7 T_{0}^{\prime \prime \prime} \rho_{0}^{\prime}}{2 \rho_{0}}+\frac{21 T_{0}^{\prime \prime} \rho_{0}^{\prime \prime}}{2 \rho_{0}}\right] \\
& -\gamma d k_{0 \|||}\left(\frac{N g \rho_{0}^{\prime \prime}}{\rho_{0}}+\frac{N g^{\prime \prime} \rho_{0}^{\prime}}{\rho_{0}}+\frac{2 N g^{\prime} \rho_{0}^{\prime \prime}}{\rho_{0}}+\frac{\gamma \rho_{0}^{\prime \prime}}{\rho_{0}} \nu^{2}\right) \\
& +\gamma d k_{0 \| \mid}\left[\frac{N g \rho_{0}^{\prime \prime}}{\rho_{0}}+\frac{N g^{\prime} \rho_{0}^{\prime}}{\rho_{0}}+\frac{\gamma \rho_{0}^{\prime}}{\rho_{0}} \nu^{2}+\left(\frac{7 T_{0}^{\prime}}{p_{0}}-\frac{2 T_{0} p_{0}^{\prime}}{p_{0}^{2}}\right)\left(N g \rho_{0}^{\prime}+\gamma \rho_{0} \nu^{2}\right)\right] \frac{f(z)^{\prime}}{(f(z)-\nu)} \\
& +\gamma d k_{0 \|||}\left[-\frac{T_{0} \rho_{0}^{\prime \prime \prime}}{\rho_{0}}-\frac{7 T_{0}^{\prime} \rho_{0}^{\prime \prime}}{\rho_{0}}+\frac{3 T_{0} \rho_{0}^{\prime} \rho_{0}^{\prime \prime}}{\rho_{0}^{2}}-\frac{2 T_{0} \rho_{0}^{\prime 3}}{\rho_{0}^{3}}-\frac{35 T_{0}^{\prime 2} \rho_{0}^{\prime}}{4 p_{0}}+\frac{7 T_{0}^{\prime} \rho_{0}^{\prime 2}}{\rho_{0}^{2}}\right] \frac{f(z)^{\prime}}{(f(z)-\nu)} \\
& +3 r \gamma\left[\left(\rho_{0}^{\prime 2} T_{0}^{-1}-\rho_{0} \rho_{0}^{\prime} T_{0}^{\prime} T_{0}^{-2}+\rho_{0} T_{0}^{-1} \rho_{0}^{\prime \prime}\right)-\frac{T_{0}^{-1} \rho_{0} \rho_{0}^{\prime} f(z)^{\prime}}{(f(z)-\nu)}\right] \\
& -\nu p_{0}^{\prime \prime}+p_{0}^{\prime} \nu \frac{f(z)^{\prime}}{(f(z)-\nu)}-(f(z)-\nu)\left(N g \rho_{0}^{\prime}+\gamma \rho_{0} \nu^{2}\right)
\end{aligned}
$$

Here all quantities are dimensionless. The prime indicates derivatives with respect to $z$.

\section{References}

Abedini, A., Safari, H.: 2011, New Astron. 16, 317.

Andries, J., Van Doorsselaere, T., Roberts, B., Verth, G., Verwichte, E., Erdélyi, R.: 2009, Space Sci. Rev. 149, 3.

Banerjee, D. Erdélyi, R., Oliver, R., O’Shea, E.: 2007, Solar Phys. 246, 3.

Berghmans, D., Clette, F.: 1999, Solar Phys. 186, 207.

Braginskii, S.I.: 1965, Rev. Plasma Phys. 1, 205.

Brynildsen, N., Maltby, P., Fredvik, T., Kjeldseth-Moe, O.: 2002, Solar Phys. 207, 259.

Curdt, W., Wang, T. J., Innes, D. E., Solanki, S. K., Dammasch, I. E., Kliem, B., \& Ofman, L.: 2002, In: Wilson, A. (ed.), Solar Variability: From Core to Outer Frontiers, ESA SP-506, 581.

DeForest, C.E., Gurman, J.B.: 1998, Astrophys. J. Lett. 501, L217.

De Moortel, I., Hood, A.W.: 2003, Astron. Astrophys. 408, 755.

De Moortel, I., Hood, A.W.: 2004, Astron. Astrophys. 415, 705.

De Moortel, I., Ireland, J., Walsh, R.W.: 2000, Astron. Astrophys. 355, L23.

Donnelly, G. R., Díaz, A. J., Roberts, B. 2006, Astron. Astrophys. 457, 707.

Dymova, M. V., Ruderman, M. S.: 2006, Astron. Astrophys. 459, 241.

Erdélyi, R., Verth, G.: 2007, Astron. Astrophys. 462, 743.

Erdélyi, R., Luna-Cardozo, M., Mendoza-Briceño, C.A.: 2008, Solar Phys. 252, 305.

Fathalian, N., Safari, H.: 2010, Astrophys. J. 724, 411.

Hildner, E.: 1974, Solar Phys. 35, 23.

Kliem, B., Dammasch, I.E., Curdt, W., Wilhelm, K.: 2002, Astrophys. J. Lett. 568, L61.

Macnamara, C.K., Roberts, B.: 2010, Astron. Astrophys. 515, A41.

Marsh, M.S., Walsh, R.W., De Moortel, I., Ireland, J.: 2003, Astron. Astrophys. 404, L37.

McEwan, M. P., Donnelly, G. R., Díaz, A. J., Roberts, B.: 2006, Astron. Astrophys. 460, M893.

McEwan, M. P., A. J. Díaz, Roberts, B.: 2008, Astron. Astrophys. 481, 819.

Mendoza-Briceńo, C.A., Erdélyi, R., Sigalotti, L. Di G.: 2004, Astrophys. J. 605, 493.

Ofman, L., Wang, T.: 2002, Astrophys. J. Lett. 580, L85.

Ofman, L., Romoli, M., Poletto, G., Noci, G., Kohl, J.L.: 1997, Astrophys. J. Lett. 491, L111.

ÓShea, E., Muglach, K., Fleck, B.: 2002, Astron. Astrophys. 387, 642.

Pandey, V.S., Dwivedi, B.N.: 2006, Solar Phys. 236, 127.

Priest, E. R.: 1982, Solar Magnetohydrodynamics, D. Reidel, Dordrecht, The Netherlands, ipage

Roberts, B.: 2006, Phil. Trans. Roy. Soc. A 364, 447.

Ruderman, M. S., Verth, G., Erdélyi, R.: 2008, Astrophys. J. 686, 694.

Safari, H., Nasiri, S., Sobouti, Y.: 2007, Astron. Astrophys. 470, 1111.

Sigalotti, L. Di G., Mendoza-Briceño . C.A., Luna-Cardozo, M. : 2007, Solar Phys. 246, 187. Srivastava, A. K., Dwivedi, B. N.: 2010, New Astron. 15, S8.

Taroyan, Y., Erdélyi, R., Doyle, J.G., Bradshaw, S.J.: 2005, Astron. Astrophys. 438, 713.

Verth, G. Van Doorsselaere, T., Erdélyi, R., Goossens, M.: 2007 Astron. Astrophys. 475, 341.

Wang, T.J., Solanki, S.K., Curdt, W., Innes, D.E., Dammasch, I.E.: 2002a, In: Wilson, A. (ed.), Proc. SOHO 11 Symposium, From Solar Min to Max: Half a Solar Cycle with SOHO, ESA $S P-508,465$. 
Abedini et al.

Wang, T.J., Solanki, S.K., Curdt, W., Innes, D.E., Dammasch, I.E.: 2002b, Astrophys. J. Lett. 574, L101.

Wang, T.J., Solanki, S.K., Innes, D.E., Curdt, W., Marsch, E.: 2003, Astron. Astrophys. 402, L17.

Wang, T.J., Solanki, S.K., Innes, D.E., Curdt, W.: 2005, Astron. Astrophys. 435, 753. 


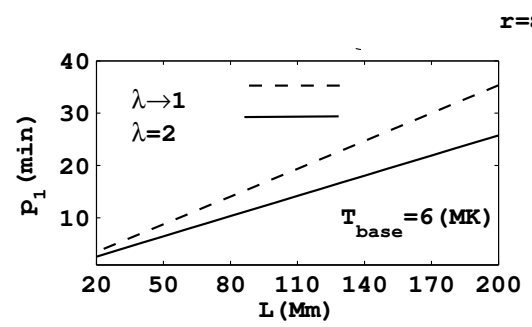

$r=\varepsilon=d=0, N \neq 0$
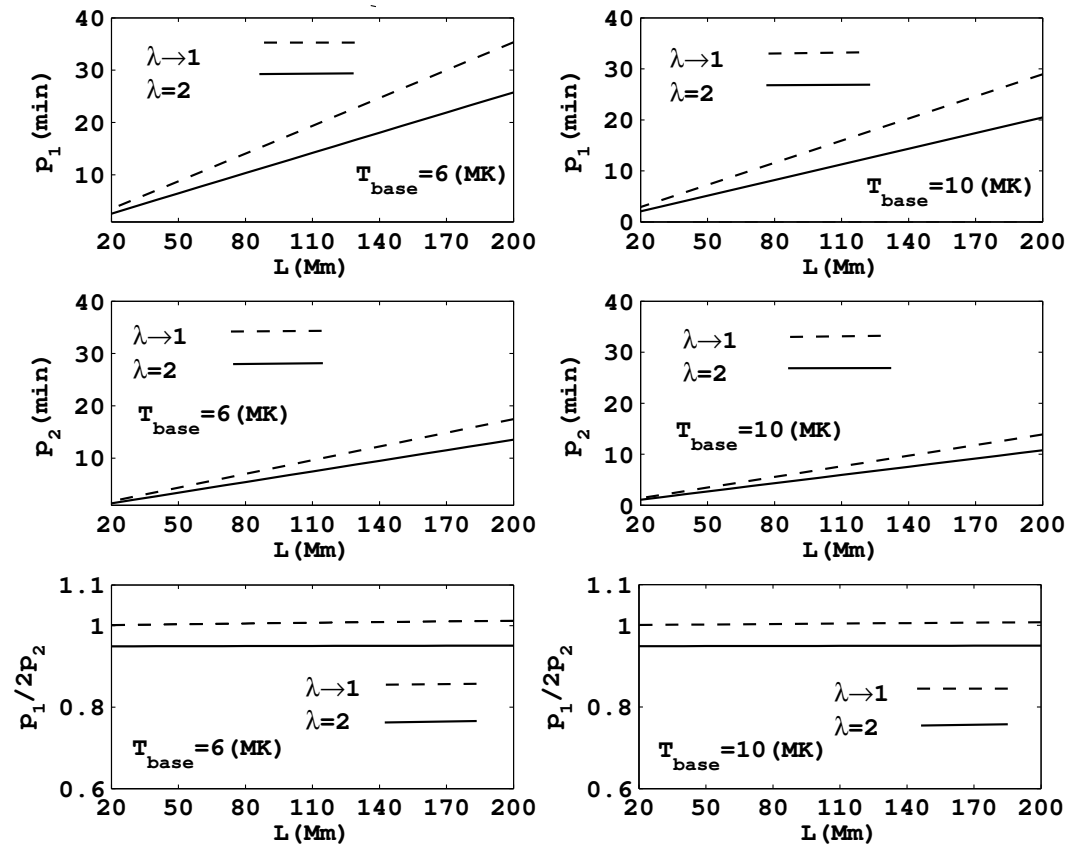

Figure 1. The periods of the fundamental mode $p_{1}$ (top row) and of the first overtone $p_{2}$ (middle row), and their ratios (bottom row) for case 1 (see text) are plotted versus $L$ in oscillating stratified loops with different values of $\lambda$ and $T_{\text {base }}$. 

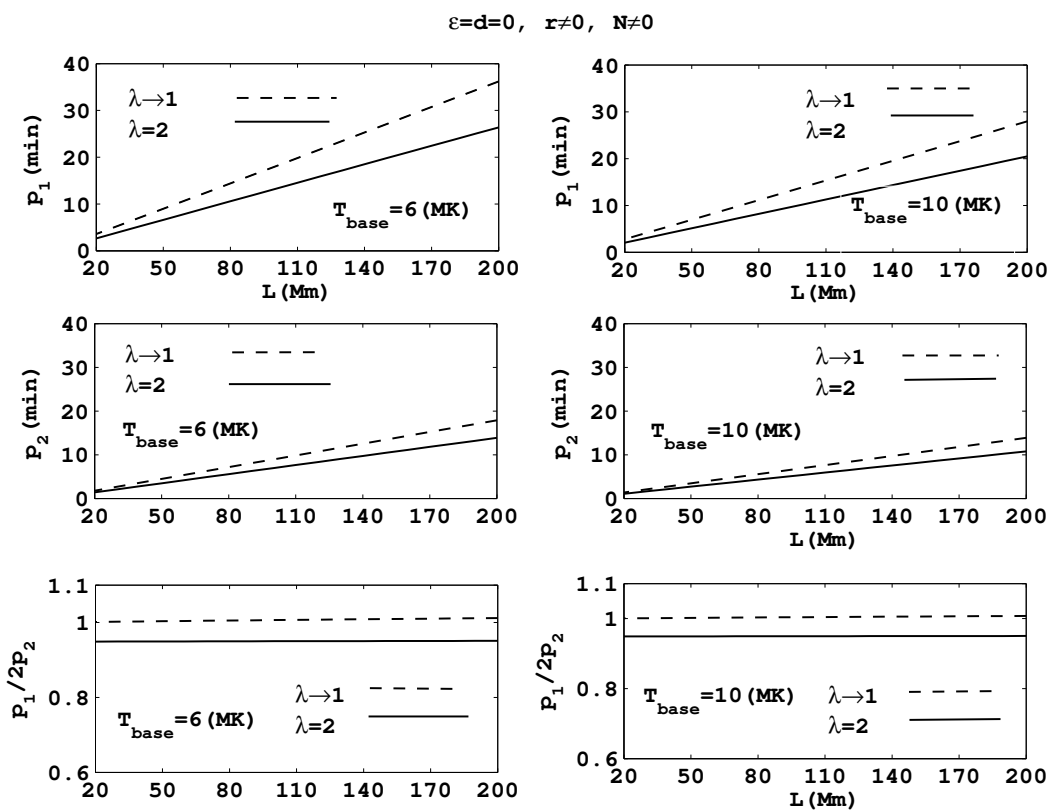

Figure 2. The periods of the fundamental mode $p_{1}$ (top row) and of the first overtone $p_{2}$ (middle row), and their ratios (bottom row) for case 2 (see text) are plotted versus $L$ in oscillating loops in the presence of radiation and gravity for different value of $\lambda$ and $T_{\text {base }}$. 

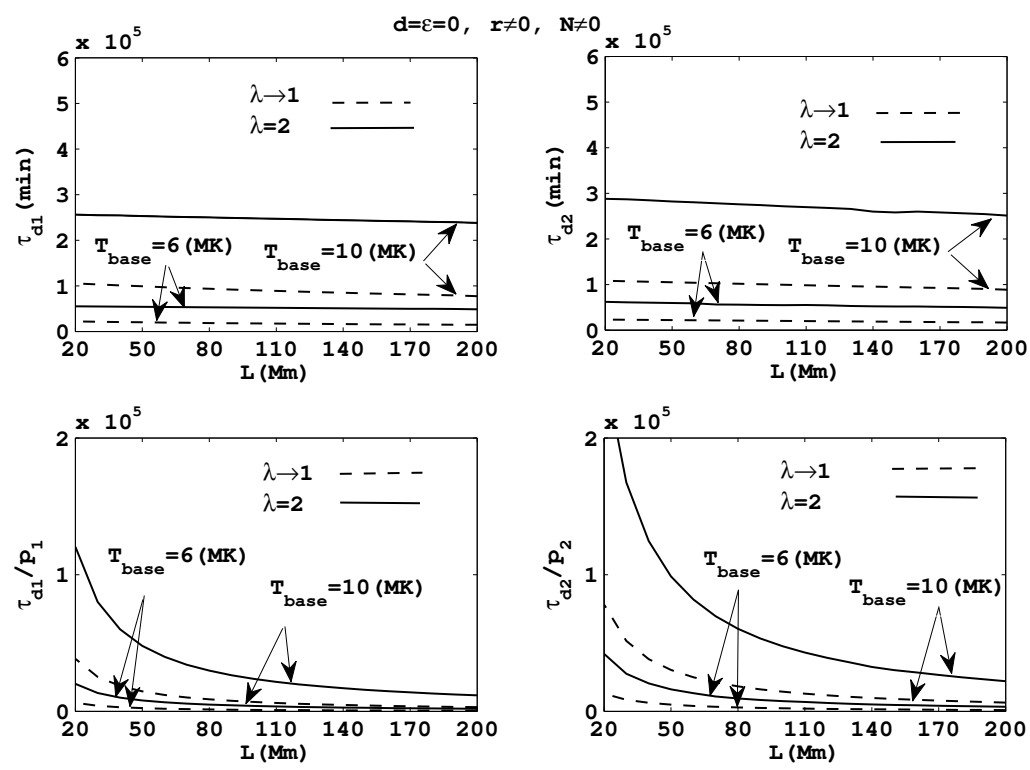

Figure 3. The damping times of the fundamental mode $\left(\tau_{\mathrm{d} 1}\right)$ and of the first overtone $\left(\tau_{\mathrm{d} 2}\right)$ (top row), and the damping times per period $\left(\tau_{\mathrm{d} 1} / p_{1}, \tau_{\mathrm{d} 2} / p_{2}\right)$ (bottom row) for case 2 (see text) are plotted versus $L$ in oscillating loops in the presence of radiation and gravity for different value of $\lambda$ and $T_{\text {base }}$. 

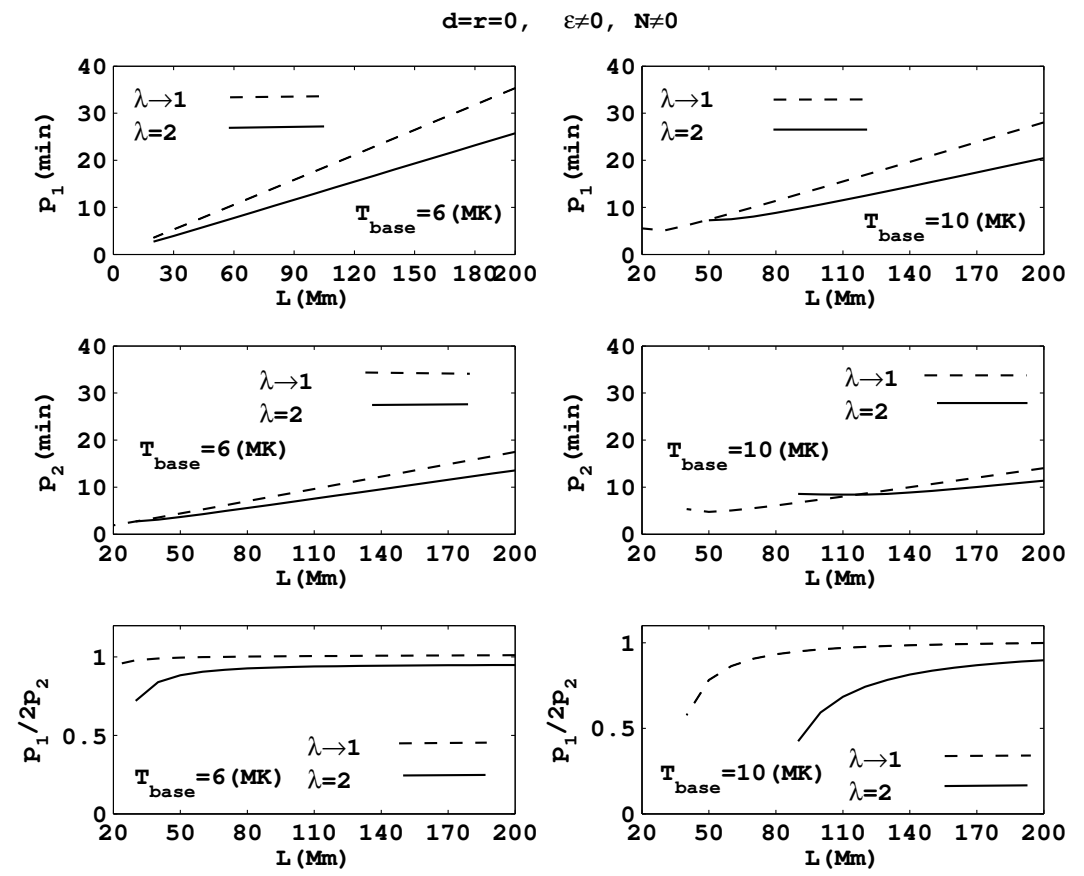

Figure 4. The periods of the fundamental mode $p_{1}$ (top row) and of the first overtone $p_{2}$ (middle row), and their ratios (bottom row) for case 3 (see text) are plotted versus $L$ in oscillating loops in the presence of viscosity and gravity for different value of $\lambda$ and $T_{\text {base }}$. 

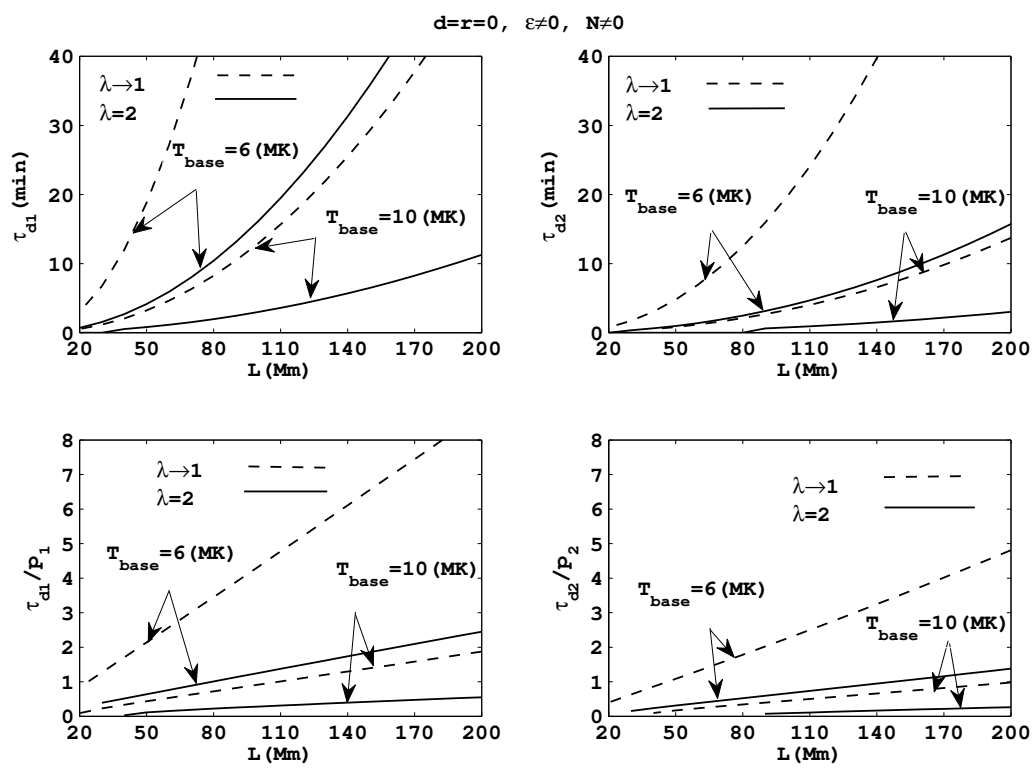

Figure 5. The damping times of the fundamental mode $\left(\tau_{\mathrm{d} 1}\right)$ and of the first overtone $\left(\tau_{\mathrm{d} 2}\right)$ (top row), and the damping times per period $\left(\tau_{\mathrm{d} 1} / p_{1}, \tau_{\mathrm{d} 2} / p_{2}\right)$ (bottom row) for case 3 (see text) are plotted versus $L$ in oscillating loop in the presence of viscosity and gravity for different value of $\lambda$ and $T_{\text {base }}$. 


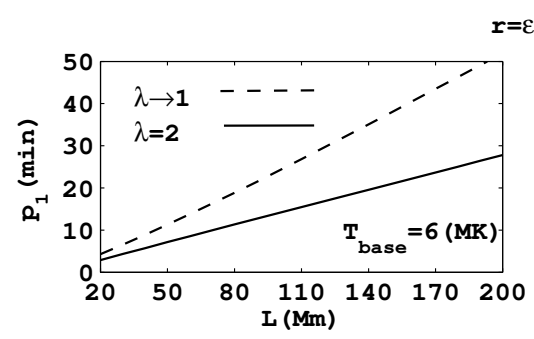

$r=\varepsilon=0, \quad d \neq 0, \quad N \neq 0$
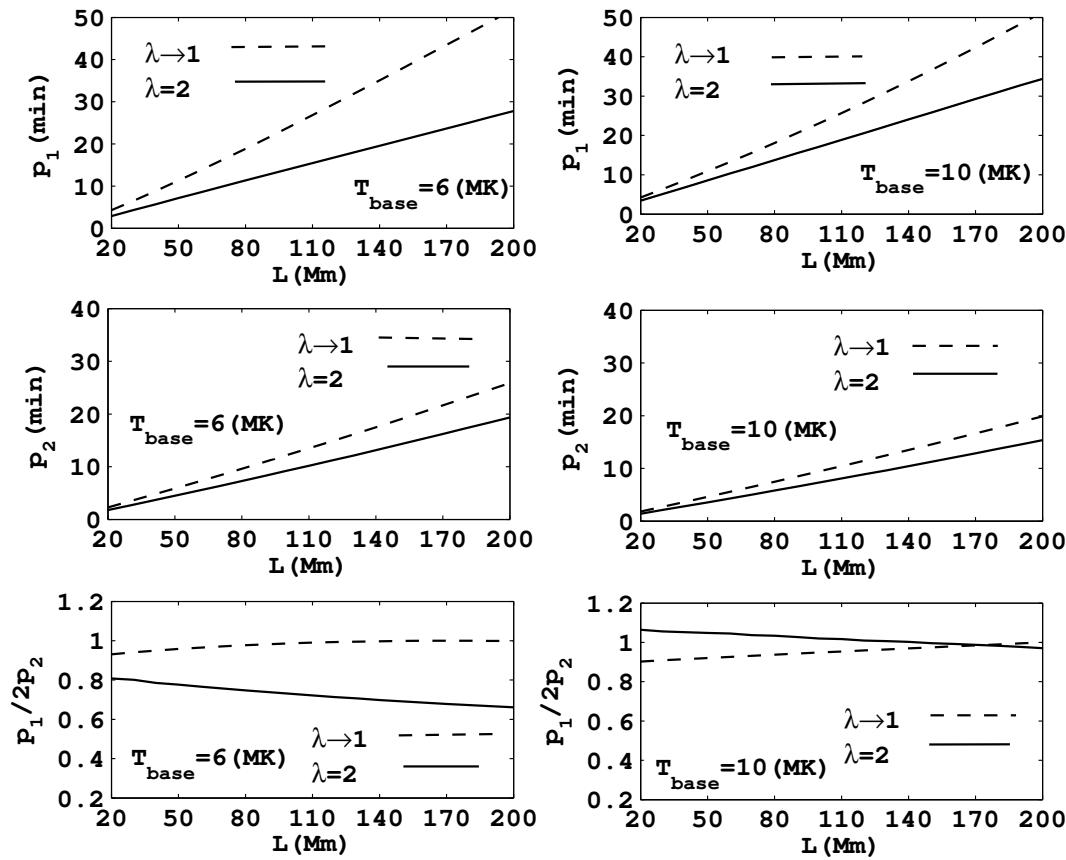

Figure 6. The periods of the fundamental mode $p_{1}$ (top row) and of the first overtone $p_{2}$ (middle row), and their ratios (bottom row) for case 4 (see text) are plotted versus $L$ in oscillating loops in the presence of conduction and gravity for different value of $\lambda$ and $T_{\text {base }}$. 


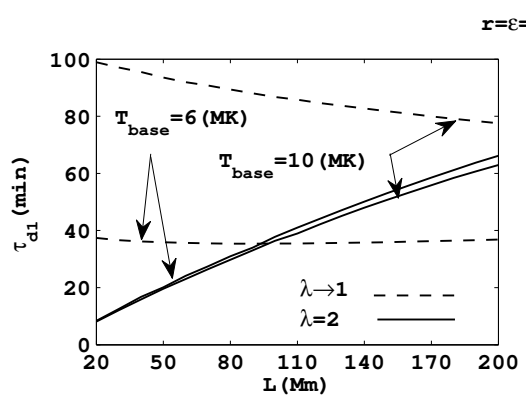

$r=\varepsilon=d \neq 0, N \neq 0$
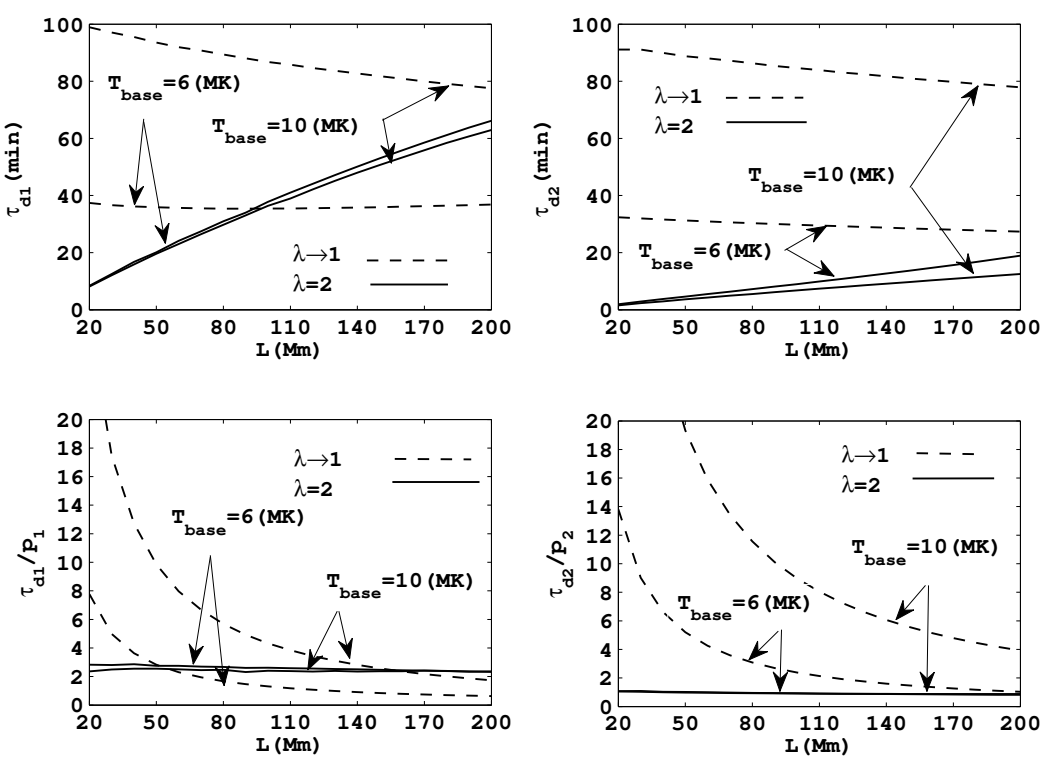

Figure 7. The damping times of the fundamental mode $\left(\tau_{\mathrm{d} 1}\right)$ and of the first overtone $\left(\tau_{\mathrm{d} 2}\right)$ (top row), and the damping times per period $\left(\tau_{\mathrm{d} 1} / p_{1}, \tau_{\mathrm{d} 2} / p_{2}\right)$ (bottom row) for case 4 (see text) are plotted versus $L$ in oscillating loops in the presence of thermal conduction and gravity for different value of $\lambda$ and $T_{\text {base }}$. 
$\mathbf{r} \neq 0, \varepsilon \neq 0, \quad d \neq 0, \quad \mathbf{N} \neq 0$
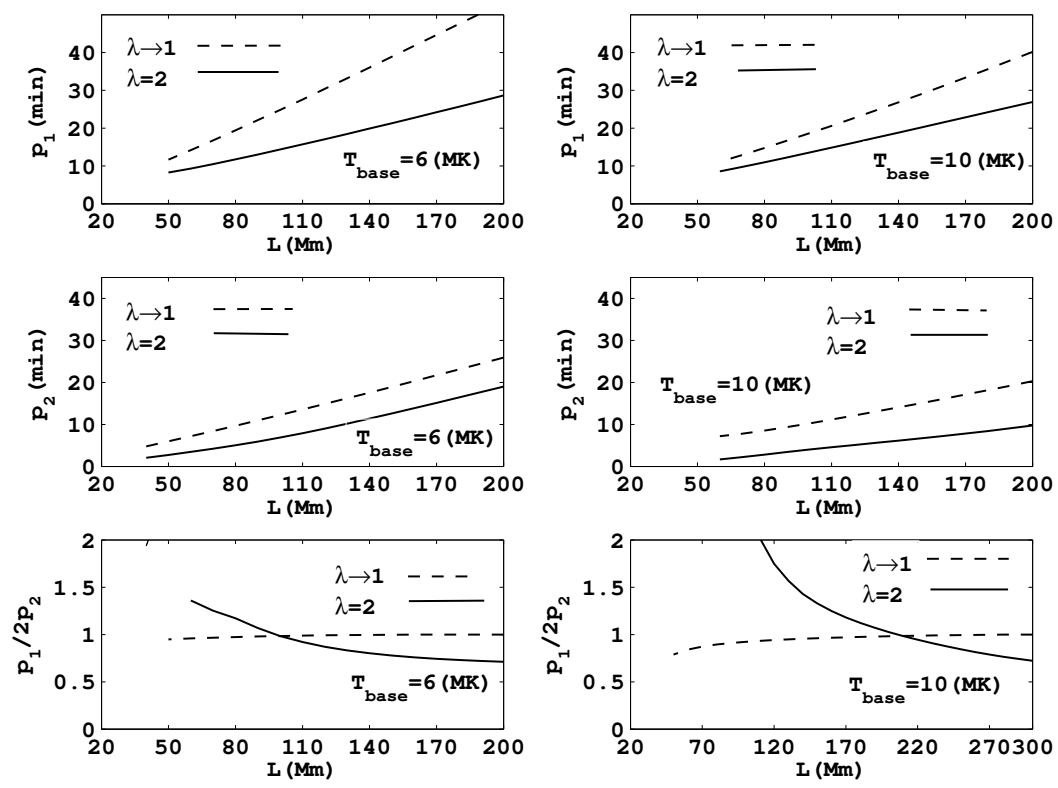

Figure 8. The periods of the fundamental mode $p_{1}$ (top row) and of the first overtone $p_{2}$ (middle row), and their ratios (bottom row) for case 5 (see text) are plotted versus $L$ in oscillating loops in the presence of radiation, viscosity, thermal conduction, and gravity for different value of $\lambda$ and $T_{\text {base }}$. 

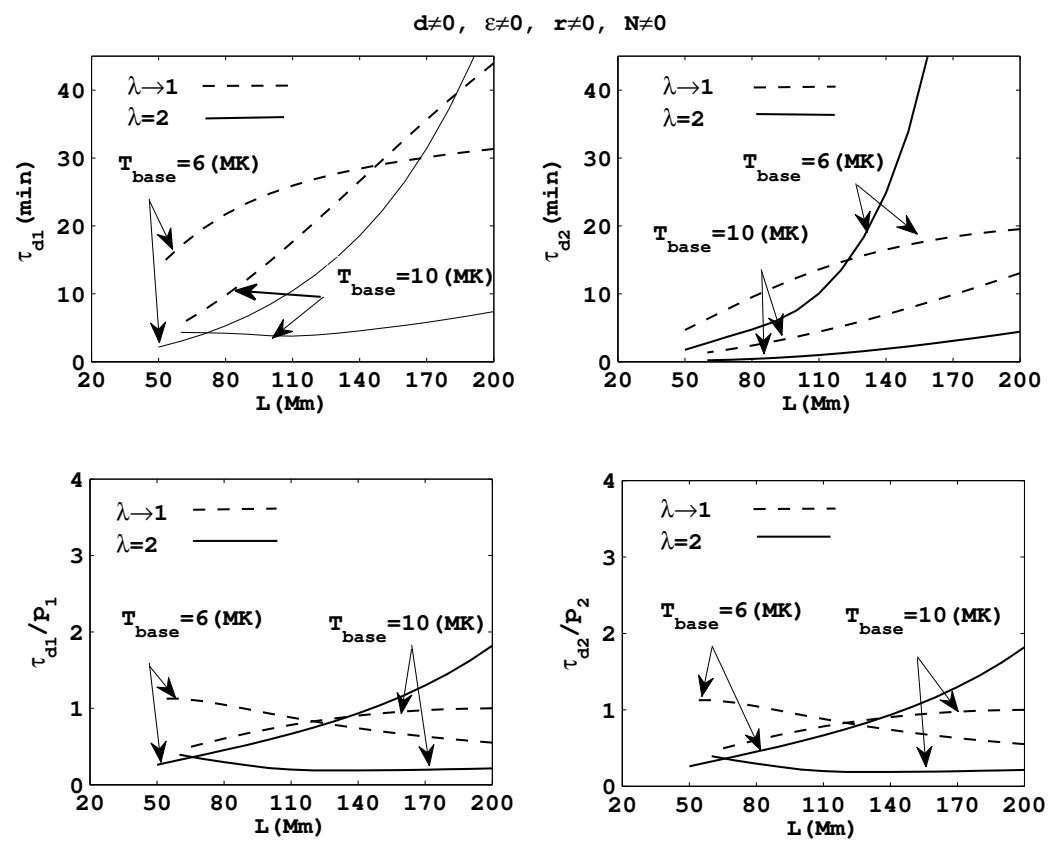

Figure 9. The damping times of the fundamental mode $\left(\tau_{\mathrm{d} 1}\right)$ and of the first overtone $\left(\tau_{\mathrm{d} 2}\right)$ (top row), and the damping times per period $\left(\tau_{\mathrm{d} 1} / p_{1}, \tau_{\mathrm{d} 2} / p_{2}\right)$ (bottom row) for case 5 (see text) are plotted versus $L$ in oscillating loops in the presence of radiation, viscosity, thermal conduction, and gravity for different value of $\lambda$ and $T_{\text {base }}$. 
SOLA1911_rev1.tex; 15/06/2018; $17: 48 ;$ p.20 